\title{
Diagnosis and treatment of accessory breast cancer in 11 patients
}

\author{
SHUO ZHANG ${ }^{1,2^{*}}$, YONG-HUA YU ${ }^{2}$, WEI QU ${ }^{2}$, YONG ZHANG ${ }^{2 *}$ and $\mathrm{JIA} \mathrm{LI}^{2}$ \\ ${ }^{1}$ School of Medical and Life Sciences, Shandong Academy of Medical Sciences, Jinan University, Jinan, Shandong 250200; \\ ${ }^{2}$ Department of Radiation Oncology, Shandong Cancer Hospital and Institute, Jinan, Shandong 250117, P.R. China
}

Received August 21, 2014; Accepted May 8, 2015

DOI: $10.3892 / \mathrm{ol} .2015 .3388$

\begin{abstract}
The present study aimed to investigate the clinical characteristics, diagnosis and treatment of accessory breast cancer, and contribute valuable information regarding this rare tumour to the current literature, ultimately facilitating the development of improved treatment strategies. The present study reported the cases of 11 patients with accessory breast cancer. The patients with accessory breast cancer were admitted between January 2002 and June 2014, and the patient records were retrospectively analysed. All patients presented with a tumour that was localised in the axilla. Out of these patients, there were 8 patients with invasive ductal carcinoma and 3 patients with invasive lobular carcinoma. The follow-up periods for patients ranged between 4 and 54 months. Out of the 5 patients that experienced neoplasm metastases, 4 patients succumbed to the disease. In total, 6 patients remain alive with no evidence of disease. Accessory breast cancer is a progressive tumour, and long-term follow-up is required. A comprehensive treatment strategy may be an effective treatment option for patients; however, the optimal time at which to commence chemotherapy and the role of combined radiotherapy and endocrine therapy require additional investigation.
\end{abstract}

\section{Introduction}

Primary carcinomas of ectopic breast tissue have been reported in only a small number of cases and the axilla was the most frequent site of the primary tumor (1). Evans et al (2) reported that $71 \%$ of ectopic breast cancers were located in the axilla. Marshall et al (3) reported that $58 \%$ occurred in the axilla, $18.5 \%$ in the parasternal, $8.6 \%$ in the subclavicular, $8.6 \%$ in the submammary, and $4 \%$ in the vulvar; $94.7 \%$ of patients were women, and only $5.3 \%$ were men. Breast tissues develop

Correspondence to: Mr. Yong-Hua Yu, Department of Radiation Oncology, Shandong Cancer Hospital and Institute, 440 Ji Yan Road, Jinan, Shandong 250117, P.R. China

E-mail: sdyonghuayu@163.com

*Contributed equally

Key words: accessory breast neoplasm, diagnosis, treatment, prognosis from the ectodermal ridges, also known as the milk lines, on the ventral surface of the body, which extend from the axillae to the inguinal regions and end on the medial aspect of the thighs on each side of the body (4). Embryologically, ectopic breast tissue develops as a result of failed resolution of the mammary ridge, an ectodermal thickening that extends from the axilla to the groin (5). Ectopic breast tissue may appear at any site along the milk line, but it occurs most commonly in the axill; less commonly, it may appear in locations outside of the mammary ridge, including the face, middle back, buttock, posterior neck, chest, vulva, hip, posterior, flank and/or lateral thigh, shoulder and upper extremities $(4,6)$.

The diagnostic procedures and therapeutic management of accessory breast carcinoma are not definitively established. The present study aimed to perform an analysis of a series of patients with accessory breast cancer that were treated exclusively with combination chemotherapy and radiotherapy, as well as to review the medical literature with regard to the clinical features, treatment methods and prognosis of this disease. The present study reports 11 such cases, with the goal of contributing valuable information about this unusual tumour to the current literature.

\section{Materials and methods}

Patients. The records of 11 patients treated for accessory breast cancer between January 2002 and June 2014 at Shandong Cancer Hospital and Institute (Jinan, Shandong, China) were retrospectively evaluated. The diagnosis for all patients was histologically confirmed by biopsies. All clinical data were obtained by reviewing the patients' medical records and communicating with the identified patients through telephone conversations and outpatient follow-up visits prior to June 2014. The follow-up period was defined as the duration between the date of the definitive diagnosis and the final visit. Written consent was obtained from all patients and the study was approved by the Ethics Committee of Shandong Cancer Hopsital and Institute.

Clinical features. Out of the 11 patients, 5 initially noticed a small subcutaneous nodule in the left axillary area, while the other 6 initially noticed a nodule in the right axillary area. In addition, 8 patients $(72.8 \%)$ were premenopausal, and 3 patients $(27.2 \%)$ were post-menopausal. The duration of disease was 3-24 months, with a median time of 12 months. The tumour size, as determined by ultrasound, computed 
tomography (CT) or magnetic resonance imaging (MRI), ranged between $0.5 \times 1.0$ and $5.0 \times 6.0 \mathrm{~cm}$. The tumours were immobile and solid with irregular boundaries. Also, 1 patient presented with enlarged ipsilateral axillary lymph nodes and 7 patients presented with the complaint of a painful tumour that was localised in the axilla.

Pre-operative examination. In total, 10 patients had undergone mammography, and 4 of these patients had no mammogram-detectable abnormalities. In the remaining patients, mammograms identified left breast lesions considered to be cancer in 3 patients and right breast cancer lesions considered to be cancer in another 3 patients. Breast and axillary ultrasonography was performed in 9 patients, which identified enlarged right axillary lymph nodes in 3 patients, right axillary accessory breast tumours in 3 patients, left axillary accessory breast tumours with axillary lymph node enlargement in 2 patients, and extremely hypoechoic nodules of the left breast with left axillary hypoechoic nodules in 1 patient. CT scans were performed on 6 patients (Fig. 1), with 2 patients demonstrating post-operative breast changes, 2 patients possessing right breast nodules, and 2 patients being considered to possess right occult breast cancer with right axillary lymph node metastasis. MRI examinations were performed on 4 patients, with 1 patient exhibiting post-operative breast changes, 2 patients demonstrating left axillary lymph node enlargement, and 1 patient being considered to possess a right accessory breast tumour. In 2 patients, positron emission tomography (PET)/CT demonstrated no evidence of any malignant or occult primary lesions except for the axillary tumour. Axillary tumour excision biopsy was performed in 3 patients, and pathological examination confirmed the diagnosis of accessory breast cancer. Axillary mass fine needle aspiration biopsies were performed on 8 patients, and cytological examination revealed cancer cells in all these patients.

Admitting diagnosis. According to the clinical and imaging features, 8 patients were preliminarily diagnosed with accessory breast cancer, 2 patients with breast cancer and 1 patient with ipsilateral axillary breast cancer recurrence.

Statistical analysis. SPSS 15.0 statistical software (SPSS Inc., Chicago, IL, USA) was used to compare the overall survival time, as calculated from the date of surgery, using the $\chi^{2}$ test. $\mathrm{P}<0.05$ was considered to indicate statistical significance.

\section{Results}

Patients. A summary of the clinical and laboratory data of the 11 patients with accessory breast carcinoma is presented in Tables I and II. All 11 patients were female, and the patient ages ranged between 27 and 48 years, with a median age of 38 years. The patients were followed up for a period ranging between 4 months and 4 years, 6 months. The median follow-up time was 20 months.

The patients exhibited systemic symptoms including fever, weight loss, night sweats, weakness, hepatosplenomegaly and lower limb oedema. Of the 11 reported patients, routine blood chemistry analyses included haematic cell analysis, electrolyte levels, liver function studies and haemograms, which revealed no abnormalities.

Perioperative management. Surgery was performed by a team of breast surgeons. The responsibilities and tasks of each surgeon were clearly defined prior to surgery. The breast surgeon was responsible for the ablative procedure, including tumour resection and sentinel node biopsy. Wide local excision with the removal of adjacent lymph nodes was the treatment of choice, and the breast was only removed if any suspicious nodules were palpable within the breast tissue. If a contralateral procedure was required, the procedure was performed simultaneously with the ablative procedures to minimise time in the operating theatre.

Treatment. Modified radical mastectomies were performed on 2 patients, and enlarged accessory breast resections and axillary lymph node dissection were performed on 6 patients (Fig. 2). The surgical specimen consisted of the post-excision skin, subcutaneous tissue and a portion of the thoracic wall. For 3 patients, the axillary tumour was regarded as locally advanced accessory breast cancer that was challenging to resect completely, and neoadjuvant chemotherapy was planned.

The resected tissue was frozen and examined pathologically to ensure tumour-free margins. The specimen was subsequently evaluated by routine histological methods. In order to avoid contamination, the surgical fields were kept strictly separated in cancer and non-cancer regions, including the use of separate instruments.

Post-operative treatment. All patients underwent 2-8 courses of chemotherapy following surgery. On average, six chemotherapy courses were administered (Table I). In total, 6 patients $(54.5 \%)$ were treated with the cyclophosphamide, doxorubicin and fluorouracil regimen, 2 patients $(18.1 \%)$ were treated with the cyclophosphamide, epirubicin and fluorouracil regimen, 1 patient $(9.1 \%)$ received the doxorubicin, cyclophosphamide and paclitaxel regimen, 1 patient $(9.1 \%)$ received the epirubicin, cyclophosphamide and paclitaxel regimen, and 1 patient (9.1\%) was treated with the cyclophosphamide, doxorubicin, fluorouracil and paclitaxel regimen. In addition, 3 patients received post-operative radiotherapy at a dose of 6,000 cGy. Following chemotherapy, 5 patients began treatment with hormone therapy, consisting of $20 \mathrm{mg}$ /day tamoxifen.

Pathological and immunohistochemical characteristics. Histological evaluation identified 8 cases $(72.7 \%)$ of invasive ductal carcinoma and 3 cases $(27.2 \%)$ of invasive lobular carcinoma. Pathological staging, which was performed according to the American Joint Committee on Cancer staging of breast cancer, revealed 2 patients with stage I disease, 4 patients with stage II disease, 4 patients with stage III disease and 1 patient with stage IV disease. Metastasis was identified in the axillary lymph nodes of 5 patients, who possessed 6 , 11, 14, 23 and 24 involved lymph nodes, respectively. In all cases, the oestrogen receptor (ER) and progesterone receptor (PR) status was determined, which revealed 9 of these tissues to be positive for ER and 7 for PR. Human epidermal growth factor receptor type 2 (HER2) status was also determined in 
Table I. Patient and disease characteristics, treatment in accessory breast carcinoma.

\begin{tabular}{ccccccccc}
\hline Patient & $\begin{array}{c}\text { Age, } \\
\text { years }\end{array}$ & Gender & $\begin{array}{c}\text { Disease } \\
\text { sites }\end{array}$ & Histopathology & Stage & $\begin{array}{c}\text { Primary } \\
\text { treatment }\end{array}$ & $\begin{array}{c}\text { Follow-up, } \\
\text { months }\end{array}$ & Metastasis \\
\hline 1 & 45 & Female & Left axilla & ILC & I & CAF & 40 & No \\
2 & 48 & Female & Right axilla & ILC & I & CEF & 54 & No \\
3 & 30 & Female & Right axilla & IDC & II & CEF & 37 & Liver \\
4 & 27 & Female & Left axilla & IDC & II & CAF & 10 & No \\
5 & 42 & Female & Left axilla & IDC & III & CAF & 13 & Axillary \\
& & & & & & & lymph node \\
6 & 38 & Female & Right axilla & IDC & III & AC-T & 4 & No \\
7 & 35 & Female & Left axilla & IDC & III & CAF & 36 & Liver, bone \\
8 & 48 & Female & Left axilla & ILC & II & CAF-T & 37 & No \\
9 & 28 & Female & Right axilla & IDC & II & EC-T & 20 & No \\
10 & 46 & Female & Right axilla & IDC & IV & CAF & 5 & Liver \\
11 & 32 & Female & Right axilla & IDC & III & CAF & 16 & Brain \\
\hline
\end{tabular}

ILC, invasive lobular carcinoma; IDC, invasive ductal carcinoma; CAF, cyclophosphamide, doxorubicin, fluorouracil; CEF, cyclophosphamide, epirubicin, fluorouracil; AC-T, doxorubicin, cyclophosphamide, paclitaxel; CAF-T, cyclophosphamide, doxorubicin, fluorouracil, paclitaxel; EC-T, epirubicin, cyclophosphamide, paclitaxel.

Table II. Clinical characteristics and laboratory examinations of accessory breast carcinoma $(\mathrm{n}=11)$.

\begin{tabular}{lc}
\hline Characteristic & Incidence, $\%$ \\
\hline Systemic symptoms & \\
Fever & 36.3 \\
Weak & 54.5 \\
Weight loss & 45.4 \\
Mass & 100 \\
Nipple discharge & 27.2 \\
Edema & 45.4 \\
Receptor expression & 11 \\
ER(+) & 81.8 \\
PR(+) & 63.6 \\
HER2(+) & 63.6 \\
\hline
\end{tabular}

ER, oestrogen receptor; PR, progesterone receptor; HER2, human epidermal growth factor receptor type 2 .

all cases, and 4 patients did not express HER2. The proliferation factor Ki67 was measured in 4 cases, all of which were expressed Ki67.

Outcome. In 7 of the reported cases (64\%), a local relapse or metastasis of the neoplasm occurred following excision (Table I). In total, 2 patients experienced recurrence and had succumbed to accessory breast cancer, with 1 patient succumbing at 20 months and the other at 40 months. Also, 1 stage III patient experienced axillary lymph node metastasis within 1 year following surgery, 1 stage III patient experienced brain metastasis within 1 year following surgery and another stage III patient experienced extensive tumour metastasis within 2 years of surgery. In addition, 1 stage II patient experienced liver recurrence $\sim 2$ years following surgery and one stage IV patient experienced liver recurrence within 4 months of tumour excision. The 2-year actuarial distant metastases rate was $36.4 \%$ (4/11). Out of the 5 patients that experienced metastasis, 4 patients succumbed subsequent to the initial diagnosis due to distant metastases. The other patient was alive with the disease at the end of follow-up. The remaining 4 patients were all alive at the end of follow-up. Patient survival duration ranged between 5 and 54 months, with a median survival time of 20 months. The three-year overall survival rate was $54.5 \%$ $(6 / 11)$.

\section{Discussion}

Breast tissue generally develops along the embryonic mammary ridge, also termed the milk line, which extends from the axilla to the groin area and appears in the sixth week of gestation (6,7). The milk line normally disappears, with the exception of the mammary ridge of the thoracic region, where normal breast tissue develops. However, accessory mammary tissue may develop due to an incomplete embryological regression of the mammary ridge, which is composed of a portion of the galactic band that runs from the axilla to the groin (5). The presence of accessory breast tissue is uncommon, with an overall incidence range of 0.22 to $6.00 \%$ in the general population $(8,9)$. Women are reported to exhibit a increased incidence of accessory breast tissue compared with men. The most common location of ectopic breast tissue is the axilla. Other less common locations are the face, thighs, perineum, groin, vulva and shoulders $(10,11)$. Ectopic breast tissue may consist of the breast parenchyma, areola, nipple or any combination of these three components (12). This breast tissue is subject to hormonal influences and undergoes physiological changes (13). In addition, a number of different neoplasms, benign and malignant, have been found in axillary breast 


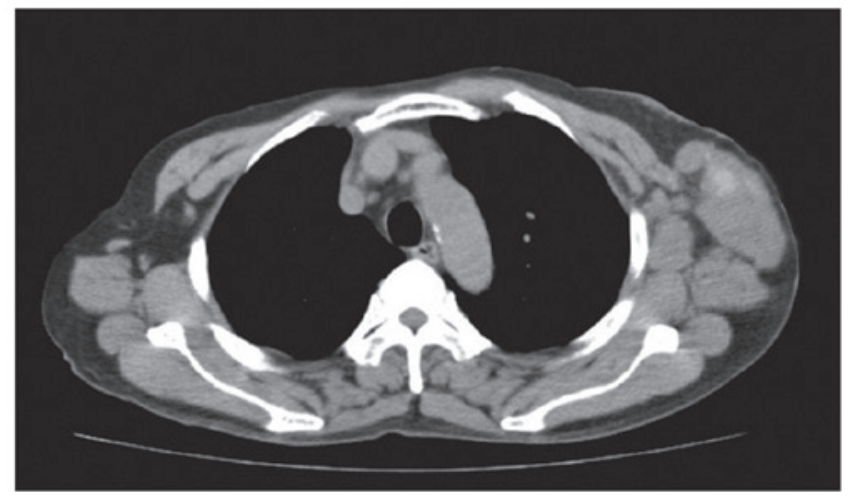

Figure 1. Computed tomography revealing enlarged lymph nodes and an exposed and lobulated soft tissue mass with faint calcification in wide contact with the skin in the left axilla.

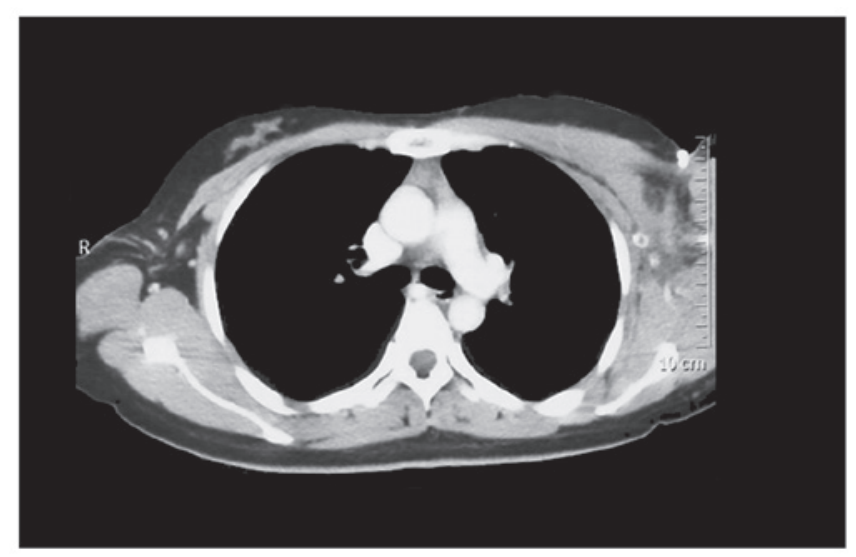

Figure 2. Computed tomograpy demonstrating the imaging manifestations of post-operative accessory breast carcinoma.

tissue (14). The most common of these tumours reported in the literature is the fibroadenoma, and there are scattered case reports of other tumours, including phyllodes tumour and mammary carcinoma (14).

Accessory mammary carcinoma is extremely rare, constituting $0.3-0.6 \%$ of all breast cancer cases, generally occurring as an axillary tumour (15). Primary breast carcinoma arising in accessory breast tissue of the axilla is the most common clinical presentation, comprising $60-70 \%$ of all ectopic breast tumours $(16,17)$. The most common pathology of accessary breast tissue carcinoma, as with normal breast carcinoma, is invasive ductal carcinoma (18). The most common physical manifestation of this carcinoma is a palpable mass. Oedema, tenderness, breast pain and non-specific discomfort are observed less often (19).

Diagnostic procedures and therapeutic management of patients with accessory breast cancer are not unequivocally established (20). The diagnosis of accessory breast cancer is not always considered and is, therefore, often delayed (21). Generally, a diagnosis of accessory breast cancer requires a pathological demonstration of cancerous tissue adjacent to normal breast ducts or lobules that are not connected to the proper mammary gland. It is also necessary to exclude the possibility of a metastatic lesion from another primary cancer (18). An accurate diagnosis of axillary accessory breast carcinoma is important, as it can provide precise staging information for patients with concurrent ipsilateral breast cancer (7). However, this diagnosis can be extremely challenging, if not impossible, since primary breast cancer frequently extends to the axillary tail and metastatic carcinoma may completely replace the involved lymph nodes (19). The differential diagnosis must include other subcutaneous masses, such as fibroadenomas, lipomas, hidradenitis and follicular cysts, as well as lymphadenopathy associated with benign or malignant disease, since aberrant breast tissue is most commonly found in the axilla (22). Therefore, axillary tail breast cancer, axillary lymph node tumour metastasis, occult breast cancer and lymphoma must be excluded prior to a firmly established diagnosis of accessory breast cancer (22).

In the current study, systemic examinations, including CT, MRI, ultrasonography and positron emission tomography/CT, revealed no primary malignant or occult lesions, only the axillary tumour. The presence of a hypoechoic, not well-defined heterogeneous mass, which is differentially diagnosed as a sebaceous cyst, without signs of inflammation, such as in hydradenitis, is considered to be accessory breast cancer (20). Mammography may contribute additional information, such as for the evaluation of the axillae, for micro-calcifications, and the breasts (23). In the case of a suspicious lesion, fine-needle aspiration biopsy or core biopsy of the axillary mass should be performed to harvest cells or tissue for histological diagnosis (8). Since the accessory breast tissue in the present study was found in the axilla, far from the pectoral breast parenchyma, the diagnosis of accessory breast carcinoma was favoured. The identification of normal breast tissue adjacent to the invasive carcinoma in the axilla strongly suggested invasive carcinoma arising in the ectopic breast tissue. The absence of accompanying lymphoid tissue further disfavoured a diagnosis of tumour metastasis in the axillary lymph node from breast cancer. Notably, accessory breast cancer may be macroscopically similar to a lymph node with metastatic carcinoma (20). Therefore, histological examination should be performed for all tissue nodules in the axilla.

Similarly to primary breast cancer, accessory breast cancer is treated surgically, which is supplemented with pre- or post-operative chemotherapy, radiotherapy and endocrine therapy. It is controversial as to whether the breast located on the same side as the accessory breast cancer should be removed as well $(22,24)$. Certain studies have recommended radical mastectomy of the ipsilateral breast if the regional lymph nodes are diagnosed with carcinoma $(11,24)$. However, Cogwells and Czerny reported that ipsilateral mastectomy does not result in an improved prognosis for patients with ectopic breast carcinoma (25). Evans and Guyton suggested that radical or modified radical surgery offered no advantage over local excision combined with axillary dissection or radiation with respect to outcome (2). It has been proposed that the surgical procedure of choice for ectopic breast carcinoma involves a wide resection of the tumour with surrounding tissue, including the skin and regional lymph nodes $(3,26)$. Mastectomy is not indicated if clinical examination, mammography and ultrasonography of the anatomic breast reveal no signs of disease, but should be 
performed when differential diagnosis is challenging $(17,27)$. If mastectomy is not performed, particularly careful follow-up is necessary to exclude any later manifestations of an occult primary neoplasm in the breast.

The present study hypothesizes that, when the accessory breast tissue is closely connected to normal breast tissue, the indications for surgery should be the same as those for a tumour anatomically situated in the breast. By contrast, when the accessory gland constitutes a separate anatomical structure, the resection of normal breast tissue appears to be unnecessary. There have been few studies on neoadjuvant chemotherapy as a treatment for locally advanced accessory breast cancer $(20,28)$. Chemotherapy with anthracycline and taxane is generally recommended for the treatment of locally advanced accessory breast cancer, as it is for the usual form of breast cancer (7). The principles of post-operative treatment are also the same as those for normal breast carcinoma (9). External radiotherapy of the tumour site must be performed to enable increased local control. However, radiation of the homolateral anatomic breast is not systematically performed (29). In the present study, 3 patients were administered with radiation therapy subsequent to surgery, while the safety and predictability have not yet been elucidated. In addition, there is a lack of evidence assessing the middle- and long-term efficacy of radiation therapy. Systemic adjuvant therapy is more frequently required due to the common occurrence of lymph node disease and is performed following the same guidelines as those used for anatomic breast carcinoma treatment (17).

In breast cancer, homolateral axillary and internal mammary nodes are considered possible sites of lymphatic metastasis (5). The lymphatic spread of axillary breast cancer is likely to only occur towards the homolateral axillary nodes and then towards the supraclavicular nodes, as this is the normal lymphatic drainage pathway of the subcutaneous and cutaneous tissues of the armpit (8). It has been reported that $37 \%$ of cases occurred prior to the age of 45 years, and due to early metastatic spread, demonstrated an even higher incidence of lymph node involvement (59-88\%) compared with primary breast carcinoma located in the external superior sector (51\%) (7). The prognosis of accessory breast carcinoma is challenging to establish, primarily due to absent or limited follow-up data as well as small sample sizes. In addition, it is challenging to identify a clear histopathological and clinical distinction between accessory and normal breast carcinoma (23). Certain studies have hypothesized that accessory breast tissue is more prone to malignancy compared with the normal breast parenchyma $(30,31)$. Marshall et al reported that the poorer outcomes in ectopic breast cancer relative to general breast cancer reflected less effective clinical disease management compared with the disease process itself (3). Certain studies have reported that the outcomes of ectopic breast cancer are poorer than those of normal breast cancer as the tumour is located near the axillary lymph nodes and is therefore associated with early metastasis to these nodes (32).

Follow-up data is provided for a limited number of patients, indicating a four-year post-treatment survival of 9.4\%, which has been calculated in a meta-analysis (2). At the time of diagnosis, the majority of patients with accessory breast carcinoma were in an advanced clinical stage, with nodal metastases or an unresectable tumour due to size $(20,28)$. Thus, the prognosis of accessory breast cancer may be worse compared with the prognosis of breast cancer arising in the proper mammary gland, although no long-term follow-up data regarding the prognosis of accessory breast cancer are available (15).

The present study reported the cases of 11 patients with rare breast carcinoma that arose in an accessory mammary gland. Due to the rarity of this disease and the consequential lack of clinical awareness, the administration of appropriate disease management is often delayed. The possibility of accessory mammary carcinoma should be considered even in male patients with neoplasms located in the axilla. The correct identification of accessory breast tissue is an important factor in the differential diagnosis of axillary tumours. Recognition of this disease by clinicians and pathologists and close communication between the two are important to prevent a misdiagnosis of malignancy and performing unnecessarily extensive surgery.

\section{References}

1. Gao YG, Zhang SH and Wang Y: A case of accessory mammary cancer in a male patient and a literature review. Eur J Gynaecol Oncol 35: 452-455, 2014.

2. Evans DM and Guyton DP: Carcinoma of the axillary breast. J Surg Oncol 59: 190-195, 1995.

3. Marshall MB, Moynihan JJ, Frost A and Evans SR: Ectopic breast cancer: Case report and literature review. Surg Oncol 3: 295-304, 1994.

4. Hao JY, Yang CC, Liu FF, et al: Accessory breast cancer occurring concurrently with bilateral primary invasive breast carcinomas: A report of two cases and literature review. Cancer Biol Med 9: 197-201, 2012.

5. Howard BA and Gusterson BA: Human breast development. J Mammary Gland Biol Neoplasia 5: 119-137, 2000.

6. Pathak S and Preston J: A rare case of multiple accessory breast tissue in the axillae, lower abdomen and vulval areas. J Obstet Gynaecol 27: 531-533, 2007.

7. Visconti G, Eltahir Y, Van Ginkel RJ, Bart J and Werker PM: Approach and management of primary ectopic breast carcinoma in the axilla: Where are we? A comprehensive historical literature review. J Plast Reconstr Aesthet Surg 64: e1-11, 2011.

8. Schmidt H: Supernumerary nipples: Prevalence, size, sex and side predilection - a prospective clinical study. Eur J Pediatr 157: 821-823, 1998.

9. Scanlan KA and Propeck PA: Accessory breast tissue in an unusual location. AJR Am J Roentgenol 166: 339-340, 1996.

10. Basu S, Bag T, Saha SK and Biswas PC: Accessory breast in the perineum. Trop Doct 33: 245, 2003.

11. Chan NG, Penswick JL, Labelle E and Driman DK: Ectopic breast tissue presenting as an anal polyp. Can J Surg 50: E23-E24, 2007.

12. Page RN, Dittrich L, King R, Boulos F and Page DL: Syringomatous adenoma of the nipple occurring within a supernumerary breast: A case report. J Cutan Pathol 36: 1206-1209, 2009.

13. Yoon HJ, Sung SH, Moon BI and Kim BS: Invasive ductal carcinoma arising from dense accessory breast visualized with $99 \mathrm{mTc}-\mathrm{MIBI}$ breast-specific $\gamma$ imaging. Clin Nucl Med 39: 717-720, 2014.

14. Devine C, Courtney CA, Deb R and Agrawal A: Invasive lobular carcinoma arising in accessory breast tissue. World J Surg Oncol 11: $47,2013$.

15. Yamamura J, Masuda N, Kodama Y, Yasojima H, Mizutani M, Kuriyama K and Sekimoto M: Male breast cancer originating in an accessory mammary gland in the axilla: A case report. Case Rep Med 2012: 286210.

16. Amsler E, Sigal-Zafrani B, Marinho E and Aractingi S: Ectopic breast cancer of the axilla. Ann Dermatol Venereol 129: 1389-1391, 2002 (In French).

17. Youn HJ and Jung SH: Accessory breast carcinoma. Breast Care (Basel) 4: 104-106, 2009.

18. Yerra L, Karnad AB and Votaw ML: Primary breast cancer in aberrant breast tissue in the axilla. South Med J 90: 661-662, 1997.

19. Pardo M, Silva F, Jiménez P and Karmelic M: Mammary carcinoma ine ectopic breast tissue. A case report. Rev Med Chil 129: 663-665, 2001 (In Spanish). 
20. Madej B, Balak B, Winkler I and Burdan F: Cancer of the accessory breast - a case report. Adv Med Sci 54: 308-310, 2009.

21. Zhang RR, Bevan S, Sun P, Lu JZ and Peng Y: Unusual presentation of multiple fibroadenomas in bilateral breasts and axillary accessory breast. Breast Cancer (Auckl) 6: 95-99, 2012.

22. Matsuoka H, Ueo H, Kuwano H, Sugimachi K and Inokuchi K: A case of carcinoma originating from accessory breast tissue of the axilla. Gan No Rinsho 30: 387-391, 1984.

23. Nakao A, Saito S, Inoue F, Notohara K and Tanaka N: Ectopic breast cancer: A case report and review of the Japanese literature. Anticancer Res 18: 3737-3740, 1998.

24. Tjalma WA and Senten LL: The management of ectopic breast cancer - case report. Eur J Gynaecol Oncol 27: 414-416, 2006.

25. Cogswell H and Czerny EW: Carcinoma of aberrant breast of the axilla. Am Surg 27: 388-390, 1961.

26. Livesey JR and Price BA: Metastatic accessory breast carcinoma in a thoracic subcutaneous nodule. J R Soc Med 83: 799-800, 1990.

27. Markopoulos C, Kouskos E, Kontzoglou K, Gogas G, Kyriakou V and Gogas J: Breast cancer in ectopic breast tissue. Eur J Gynaecol Oncol 22: 157-159, 2001
28. Takeyama H, Takahashi H, Tabei I, Fukuchi O, Nogi $\mathrm{H}$ Kinoshita S, Uchida K and Morikawa T: Malignant neoplasm in the axilla of a male: Suspected primary carcinoma of an accessory mammary gland. Breast Cancer 17: 151-154, 2010.

29. Routiot T, Marchal C, Verhaeghe JL, Depardieu C, Netter E, Weber B and Carolus JM: Breast carcinoma located in ectopic breast tissue: A case report and review of the literature. Oncol Rep 5: 413-417, 1998

30. Ghosn SH, Khatri KA and Bhawan J: Bilateral aberrant axillary breast tissue mimicking lipomas: Report of a case and review of the literature. J Cutan Pathol 34 (Suppl 1): 9-13, 2007.

31. Francone E, Nathan MJ, Murelli F, Bruno MS, Traverso E and Friedman D: Ectopic breast cancer: Case report and review of the literature. Aesthetic Plast Surg 37: 746-749, 2013.

32. Copeland MM and Geschickter CF: Diagnosis and treatment of premalignant lesions of the breast. Surg Clin North Am 30: 1717-1741, 1950. 